\title{
Modules and Front-End Electronics Developments for the ATLAS ITk Strips Upgrade
}

\author{
Carlos García Argos, on behalf of the ATLAS ITk Collaboration \\ University of Freiburg (Germany) carlos.garcia.argos@cern.ch
}

\begin{abstract}
The ATLAS experiment is currently preparing for an upgrade of the tracking system in the course of the High Luminosity LHC, scheduled for 2024. The existing Inner Detector will be replaced by an all-silicon Inner Tracker (ITk) consisting of a pixel detector surrounded by a strip detector. The ITk strip detector consists of a four layer barrel and a forward region composed of six discs on each side of the barrel. The basic unit of the detector is the silicon-strip module, consisting of a sensor and one or more hybrid circuits that hold the read-out electronics. To account for the different geometries of the central and forward regions, the barrel and end-cap modules have different geometries. In the central region, the detectors are rectangular with straight strips, whereas in the forward region the modules require wedge shaped sensors with varying strip length and pitch. The current prototyping phase has resulted in the ITk Strip Detector Technical Design Report (TDR). In this contribution we present the current status of R\&D of the ITk Strip Detector modules and read-out electronics.
\end{abstract}

Keywords: ATLAS ITk, strips detector, electronics

\section{Introduction}

The ATLAS Experiment [1] will replace the Inner Detector by an all-silicon Inner Tracker, named ITk. This will allow ATLAS to cope with the High Luminosity LHC conditions: tenfold increase in pile-up and radiation with respect to the current LHC, for a maximum fluence of $1.2 \times 10^{15} \mathrm{n}_{\mathrm{eq}} / \mathrm{cm}^{2}$ and 50 MRad ionising dose. At the same time, power consumption and material have to be kept low in order to not degrade the physics performance of ATLAS.

In the ITk the number of read-out channels will increase from 80 million to about 600 million in the pixel detector and from 6 million to about 70 million in the strips detector. The strips detector will replace both the current Semiconductor Tracker (SCT) and the Transition Radiation Tracker (TRT).

We discuss some of the design choices and results on the current prototyping status of the strips detector modules, with emphasis on the Technical Design Report [2] published earlier this year. 


\section{The ATLAS ITk Strips Detector}

The ITk strips detector will consist of four barrel layers and six end-cap discs on each side of the barrel. The barrel cylinders will be made from staves, which are a straight assembly of rectangular modules. The end-cap discs will be made from petals, which are an assembly of different modules with a wedge shape.

Both staves and petals are double-sided for two dimensional resolution and have integrated services: cooling, power and data read-out.

The total power of the tracker will be around $60 \mathrm{~kW}$, more than the $19 \mathrm{~kW}$ of the current SCT, where about $60 \%$ of the total power is lost in the cables due to ohmic losses [3]. Due to lack of space and material constraints, it will not be possible to add cables to reduce the losses. In order to minimise power losses, we have opted for use of DC-DC conversion at the modules, leading to lower currents on the cables. Additionally, a single high voltage cable will be used to bias multiple sensors, making use of high voltage switches for multiplexing [4].

Both the DC-DC converter and the high voltage switch are integrated on a power board that will also include control and monitoring functions of the voltages, currents and temperature of the modules.

\subsection{Silicon Strips Modules}

A module is the basic detector element, made from one or more hybrids glued on a sensor using non-conductive glue. The power board is glued on the sensor, next to the hybrid or hybrids. Wire-bonds are used to electrically connect the the ASICs to the sensor and the hybrid to the power board. Figure 1 shows an exploded view of a barrel module, with its various components and layers. The sensors are n-in-p float-zone silicon sensors with $74.5 \mu \mathrm{m}$ strip pitch and $300 \mu \mathrm{m}$ thickness. The n-in-p technology choice was made after studying the radiation hardness of different sensor types [5].

In the initial prototyping the modules are mounted on a test-frame for individual testing purposes. Power is fed from one side and data is transmitted and received from the opposite side. After this initial prototyping using test-frames, a stave is being populated and tested, with promising results.

The hybrid circuits of the strips modules are made from a polyimide base, using up to four copper layers. Multiple read-out chips are glued on the circuit, electrically connected to the circuit via wire-bonds.

\section{$2.2 \quad$ Front-end Electronics}

The read-out of the current prototypes of strips detectors is based on a set of two chips: the ATLAS Binary Chip ABC130 and the Hybrid Controller Chip (HCC130). These are ASICs designed in a $130 \mathrm{~nm}$ CMOS process.

The ABC130 chip reads out the 256 strips from a sensor at a clock rate of $40 \mathrm{MHz}$. The read-out is binary, with the outputs of the discriminators stored in a 256 bit pipeline. 


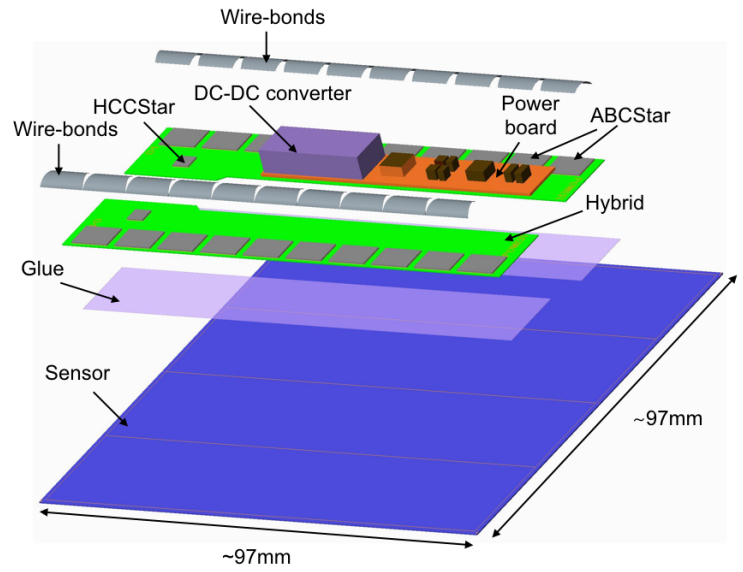

Fig. 1. Exploded view of a barrel module.

The data coming from the multiple $\mathrm{ABC} 130$ chips on a module are sent to the HCC130, which aggregates the data and interfaces to the Data Acquisition (DAQ) system. These chips employ a daisy chain read-out architecture, as shown in Fig. 2.

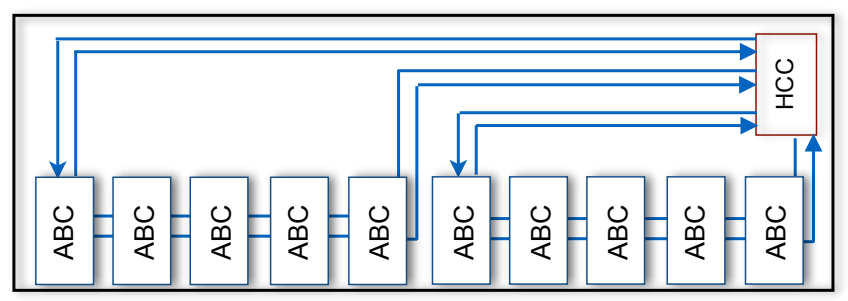

Fig. 2. Read-out architecture of the HCC130 and ABC130 chipset.

The performance of the modules and front-end electronics has been proven at various test-beams, at DESY with 4 to $4.8 \mathrm{GeV}$ electrons and at CERN with $120 \mathrm{GeV}$ pions. Both non-irradiated and irradiated modules show an adequate performance in test-beams, as shown in the efficiency plot in Fig. 3.

\subsection{The ABCStar and HCCStar Front-end Chips}

There is a final design of the front-end chips. They are named ABCStar and HCCStar, taking the "Star" name from the read-out architecture. The daisy 


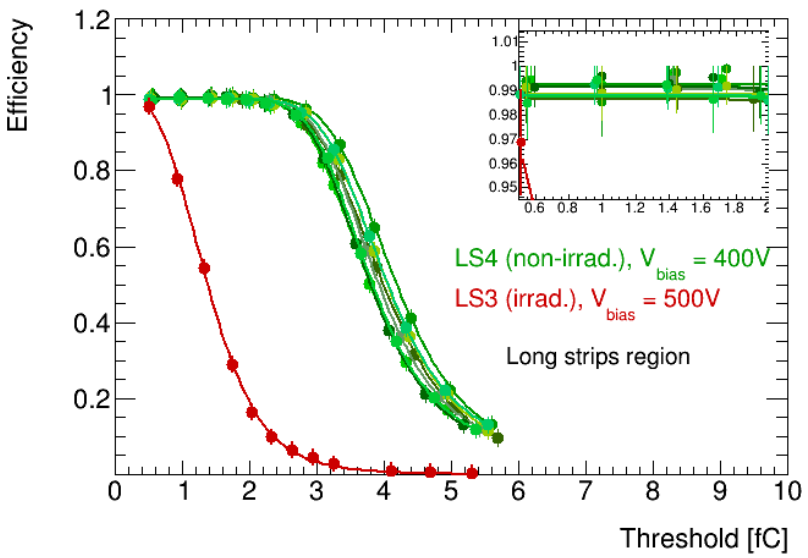

Fig. 3. Efficiency vs threshold for both irradiated and non-irradiated modules measured in a test-beam.

chain of $\mathrm{ABC}$ chips is removed and all front-end chips have a point-to-point connection to the HCCStar.

Initial tests with a prototype featuring 32 channels reveal higher initial noise of the new design before irradiation, as shown in Fig. 4. The noise is however lower for the new design after irradiation.

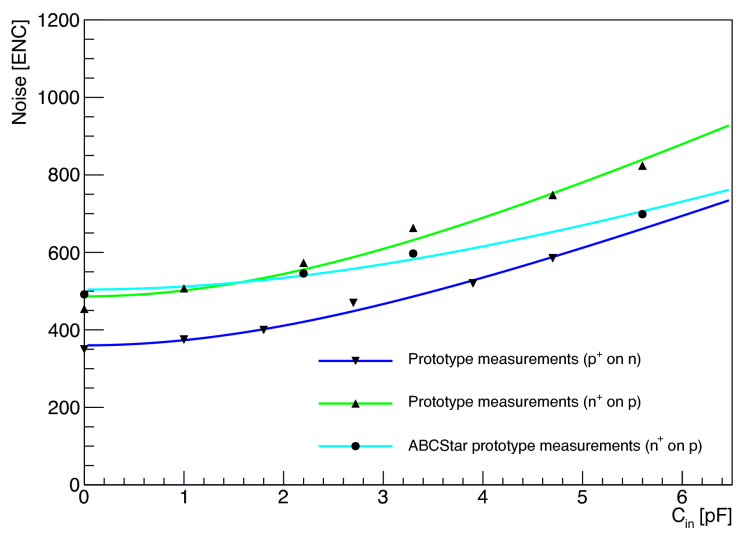

Fig. 4. Noise measurements for different input capacitance values with ABC130 and ABCStar protoypes. 


\section{Conclusions}

The prototyping phase of the ATLAS ITk Strips Upgrade is almost concluded. The final design for the front-end electronics is ready to be produced. An extensive testing programme that includes calibration measurements and test-beams, has shown that the modules have good performance even after irradiation to the expected accumulated dose at the end of the HL-LHC operation.

\section{References}

1. ATLAS Collaboration: The ATLAS Experiment at the CERN Large Hadron Collider, JINST 3 (2008) S08003.

2. ATLAS Collaboration: Technical Design Report for the ATLAS Inner Tracker Strip Detector. CERN-LHCC-2017-005, April 2017.

3. P. W. Phillips: ATLAS SCT Power Supply System. Proceedings of TWEPP 2007, CERN-2007-007, 365-368, September 2007.

4. E. G Villani et al: HVMUX, the High Voltage Multiplexing for the ATLAS Tracker Upgrade. JINST 10 (2015) C01041.

5. M. Lozano et al.: Comparison of radiation hardness of P-in-N, N-in-N, and N-in$\mathrm{P}$ silicon pad detectors. Nuclear Science, IEEE Transactions on, vol. 52, no. 5, pp. 1468-1473, 2005. 